\title{
A fast read retry method for 3D NAND flash memories using novel valley search algorithm
}

\author{
Qianhui Li ${ }^{1,2,3}$, Qi Wang ${ }^{1,2,3 a)}$, Qikang $\mathbf{X u}^{1,2,3}$, \\ and Zongliang Huo $\mathbf{H}^{1,2,3 \mathrm{~b})}$ \\ ${ }^{1}$ University of Chinese Academy of Sciences, Beijing, China \\ ${ }^{2}$ Institute of Microelectronics of Chinese Academy of Sciences, Beijing, China \\ ${ }^{3}$ Yangtze Memory Technologies Co., Ltd., Wuhan, China \\ a)wangqi1@ime.ac.cn \\ b)huozongliang@ime.ac.cn
}

\begin{abstract}
Read retry is one of the most effective methods to reduce retention errors caused by charge loss. A novel valley search algorithm for fast read retry method is proposed in this paper, which can reduce read operations to two times from at least three by conventional valley search algorithm $[1,8]$. Experimental results illustrate that raw bit error rate (RBER) after read retry is reduced to $0.0344 \%$ using the novel valley search algorithm, which is within $4.41 \%$ of $\mathrm{BCH}(9088,8192,64)$ error correcting code (ECC) $[2,3]$ error correction capability.
\end{abstract}

Keywords: NAND flash memory, read retry, valley search

Classification: Integrated circuits

\section{References}

[1] C. Kim, et al.: "A 512 Gb 3b/cell 64-stacked WL 3D V-NAND flash memory," ISSCC (2017) 202 (DOI: 10.1109/ISSCC.2017.7870331).

[2] P. Chen, et al:: "High performance low complexity BCH error correction circuit for SSD controllers," EDSSC (2015) 217 (DOI: 10.1109/EDSSC.2015. 7285089).

[3] K. Lee, et al.: "Low-cost, low-power and high-throughput $\mathrm{BCH}$ decoder for NAND Flash Memory,” ISCAS (2012) 413 (DOI: 10.1109/ISCAS.2012. 6272051).

[4] H. Maejima, et al.: “A $512 \mathrm{~Gb} 3 \mathrm{~b} / \mathrm{Cell}$ 3D flash memory on a 96-word-linelayer technology,” ISSCC (2018) 336 (DOI: 10.1109/ISSCC.2018.8310321).

[5] Y. Cai, et al:: "Data retention in MLC NAND flash memory: Characterization, optimization, and recovery," HPCA (2015) 551 (DOI: 10.1109/HPCA.2015. 7056062).

[6] N. Papandreou, et al.: "Using adaptive read voltage thresholds to enhance the reliability of MLC NAND flash memory systems," GLSVLSI (2014) 151 (DOI: 10.1145/2591513.2591594).

[7] S. C. Kang, et al.: U.S. Patent 0066899 (2011).

[8] Y. Wu, et al.: U.S. Patent 8953373-B1 (2015).

[9] Y. Cai, et al.: "Threshold voltage distribution in MLC NAND flash memory: 
Characterization, analysis, and modeling,” DATE (2013) 1285 (DOI: 10.7873/

DATE.2013.266).

[10] Y. Wu and M. Marrow: U.S. Patent 013188 (2014).

[11] C. A. Aslam, et al:: "Dynamic write-level and read-level signal design for MLC NAND flash memory," CSNDSP (2014) 336 (DOI: 10.1109/CSNDSP.2014. 6923850).

\section{Introduction}

With the development of multi-layer stack and multi-bit per cell storage technology, the storage density of 3D NAND Flash Memory has been increasing continuously in recent years. Toshiba has developed their 3b/Cell 3D Flash Memory on a 96-Word-Line-Layer technology in 2018 [4]. However, due to the increase of storage density, especially the development of multi-bit per cell storage technology, 3D NAND Flash Memory is more vulnerable to retention noise $[5,6]$. Read retry using valley search algorithm which is also called threshold voltage tracking read $[1,4]$ is one of the most effective methods to solve the problem of retention noise. The most simple and effective method of valley search is to increase or decrease read voltage at a certain interval until the optimal value of read voltage found [7]. Another efficient method has been proposed in a US patent, which finds the relative position between current read voltage and threshold voltage distribution by three read operations [8]. However, both of them have disadvantages on read retry performance, such as more read operations in one search and longer searching time.

\section{Theory of novel valley search algorithm}

The novel valley search algorithm proposed in this paper is a practical approach for fast read retry which can find the threshold voltage distribution valley by using two read operations. The raw bit error rate can be greatly reduced using optimal read voltage which is found by our novel valley search algorithm. In addition, read errors can be further corrected by Error Correction Code (ECC) with high probability. The number of read operations using by threshold voltage tracking can be reduced dramatically by using our novel valley search algorithm.

\subsection{Symbols and definitions}

Normal distribution, also named Gaussian distribution, is widely used as the model of programmed threshold voltage distribution in industrial applications and academic research $[9,10,11]$. As shown in Fig. 1, the dotted line (L1) represents initial programmed threshold voltage distribution state and programmed threshold voltage distribution state after storage time is in solid line (L2). Functional relationships between $L_{A}$ and $B, C$ will be discussed in section 2.2. Definitions and symbols being used in this paper is shown in Table I.

\subsection{Details of novel valley search algorithm}

The specific novel valley search algorithm need three steps to implement. First, the valley search algorithm proposed in this paper needs two read operations to find the 


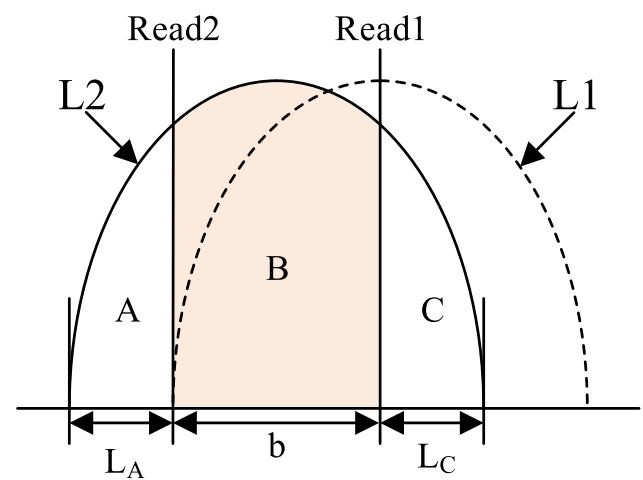

Fig. 1. Threshold voltage shift estimate model of the highest programmed threshold voltage distribution state

Table I. Symbols and definitions

\begin{tabular}{ll}
\hline$V_{T}$ & Threshold voltage \\
\hline$P_{\text {highest }}$ & The highest programmed $V_{T}$ distribution state \\
\hline$P_{1}, P_{2}, P_{3}$ & Programmed $V_{T}$ distribution state of $\mathrm{MLC}, P_{3}=P_{\text {highest }}$ \\
\hline$P_{A}, P_{B}, P_{C}$ & The probability of cells distributing in the region $\mathrm{A}, \mathrm{B}, \mathrm{C}$ \\
\hline$A, B, C$ & The number of cells distributing in the region $\mathrm{A}, \mathrm{B}, \mathrm{C}$ \\
\hline$S u m$ & The total number of cells distributing in $P_{\text {highest }}$ \\
\hline$L_{A}$ & The $V_{T}$ shift of the highest programmed $V_{T}$ distribution state \\
\hline$R e a d 1, R e a d 2$ & The fixed read voltage level using by read operation \\
\hline$b$ & The fixed voltage level $(b=R e a d 1-R e a d 2)$ \\
\hline$a$ & The quotient of $B / C$ ( $a=B / C)$ \\
\hline$f_{0}(x)$ & The probability density function of Gauss distribution, $\mu=0$ \\
\hline$F_{0}(x)$ & The cumulative distribution function of Gauss distribution, $\mu=0$ \\
\hline$v_{i}\left(L_{A}\right)$ & Threshold voltage shift of lower $V_{T}$ distribution, $\mathrm{i}=1,2, \ldots$ \\
\hline$R_{i}$ & Initial read voltage before read retry, i $=1,2, \ldots$ \\
\hline$R_{n}$ & Initial read voltage of $P_{h i g h e s t ~}$ before read retry \\
\hline$R_{E i}$ & Read voltage using by read retry operations, $\mathrm{i}=1,2, \ldots$ \\
\hline$R_{E n}$ & Read voltage of $P_{\text {highest }}$ using by read retry \\
\hline
\end{tabular}

threshold voltage shift of the highest programmed threshold voltage distribution state. Fig. 1 and Fig. 2 can help us understand this method. Second, the threshold voltage shift of lower programmed threshold voltage distribution state can be calculated by determined functional relationships between threshold voltage shift of the highest programmed threshold voltage distribution state and threshold voltage shift of lower programmed threshold voltage distribution state [4]. Finally, read voltages used by read retry operations can be calculated by initial read voltage minus threshold voltage shift. The value of $B$ and $C$ can be obtained by two read operations with different read voltage.

The value of $b$ can be calculated as:

$$
b=\operatorname{Read} 1-\operatorname{Read} 2
$$

According to the theory of probability, those mathematical formulas can be obtained: 


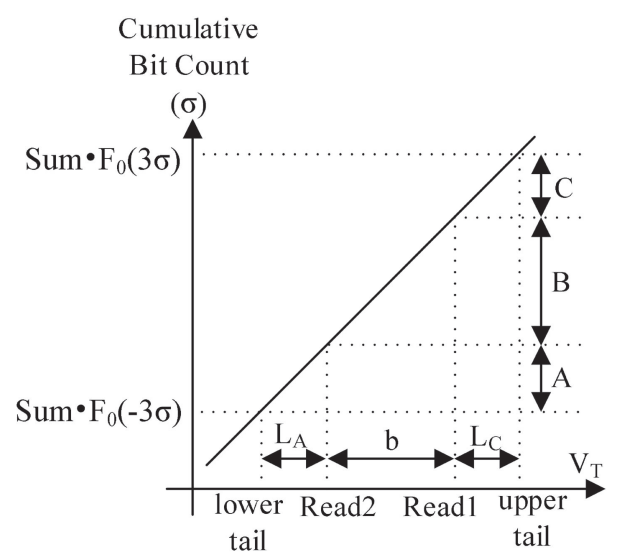

Fig. 2. Relationship between threshold voltage distribution and cumulative bit count

$$
\begin{aligned}
& P_{A}+P_{B}+P_{C}=1 \\
& \left\{\begin{array}{l}
A=\mathrm{Sum} \cdot P_{A} \\
B=\mathrm{Sum} \cdot P_{B} \\
C=\mathrm{Sum} \cdot P_{C}
\end{array}\right.
\end{aligned}
$$

According to equation (3), we have:

$$
a=B / C=P_{B} / P_{C}
$$

According to equation (2) and (4), we have:

$$
P_{A}+(a+1) P_{C}=1
$$

If $P_{\text {highest }}$ is in concordance with Gaussian distribution, we have:

$$
\left\{\begin{array}{l}
L_{A}+b+L_{C} \approx 6 \sigma \\
P_{A}=F_{0}\left(-3 \sigma+L_{A}\right)-F_{0}(-3 \sigma) \\
P_{C}=F_{0}(3 \sigma)-F_{0}\left(3 \sigma-L_{C}\right)
\end{array}\right.
$$

According to equation (6), $P_{A}$ and $P_{C}$ can be calculated as:

$$
\left\{\begin{array}{l}
P_{A}=F_{0}\left(-3 \sigma+L_{A}\right)-F_{0}(-3 \sigma) \\
P_{C}=F_{0}(3 \sigma)-F_{0}\left(-3 \sigma+L_{A}+b\right)
\end{array}\right.
$$

According to equation (5) and (7), we have:

$$
F_{0}\left(-3 \sigma+L_{A}\right)-F_{0}(-3 \sigma)+(a+1)\left[F_{0}(3 \sigma)-F_{0}\left(-3 \sigma+L_{A}+b\right)\right]=1
$$

The implicit function relationship shown in equation (8) can determine a functional relationship with two variables:

$$
L_{A}=L_{A}(B, C)
$$

Read voltages, used by read retry operations, can be determined by:

$$
\left\{\begin{array}{l}
R_{E n}=R_{n}-L_{A}(B, C) \\
R_{E i}=R_{i}-v_{i}\left(L_{A}\right)
\end{array}\right.
$$

The proposed novel valley search algorithm for NAND flash memory is listed as 


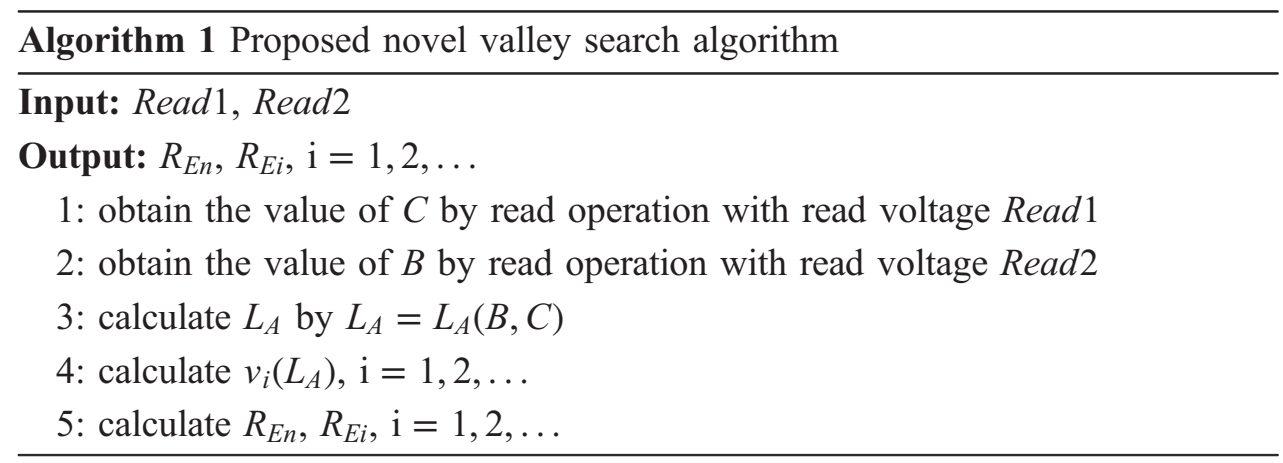

\section{Numerical solution method of mathematical model}

The relationship between $L_{A}$ and $B, C$ is described in equation (8), which can be obtained by using numerical solution method. As Fig. 3(a) shows, $L_{A}$ is singlevalued function determined by $B$ and $C$, which means one unique pair of $B$ and $C$ can determine one unique $L_{A}$. The value of $L_{A}$ determined by one unique pair of $B$ and $C$ greater than zero means threshold voltage distribution shift to the left, otherwise the value of $L_{A}$ less than zero means threshold voltage distribution shift to the right. Since Read1 and Read2 are both fixed read voltage level, $B$ and $C$ will change synchronously actually. The dotted line shown in Fig. 3 . is an actual example. When $C$ equals 0 , as Fig. 3(1) shows, the maximum of $L_{A}$ will be achieved and the maximum of $L_{A}$ is $6 \sigma-b$. When $B$ equals 0 , as Fig. 3(3) shows, the minimum of $L_{A}$ will be achieved and the minimum of $L_{A}$ is $-b$. In actual engineering application, the functional relationship (Fig. 3(a)) between $L_{A}$ and $B, C$ can be implemented as lookup tables stored in ROM.

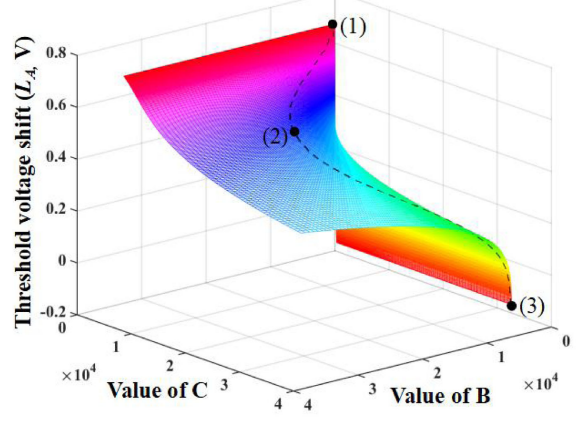

(a)

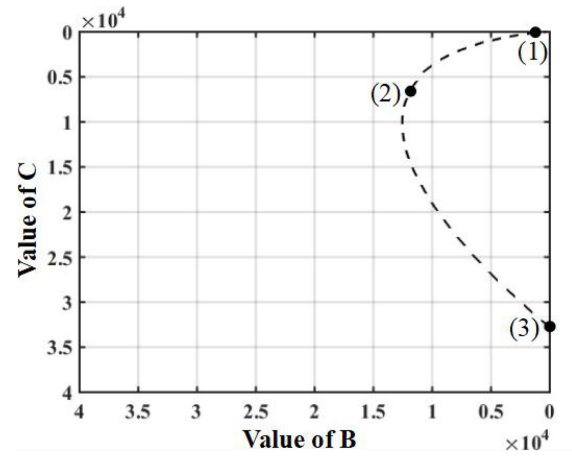

(b)

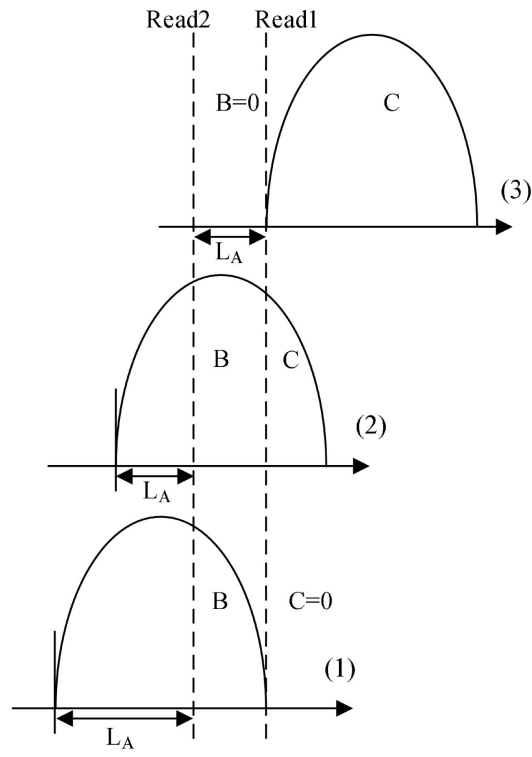

(c)

Fig. 3. Functional relationship between $L_{A}$ and $B, C$ 
An optimal read voltage of the lower states calibrating read can be calculated using the high-state threshold voltage tracking read result [4]. In order to establish the mathematical model to calculate the threshold voltage shift of lower states, MLC 3D NAND Flash Memory has been tested to find the functional relationships between threshold voltage shift of $P_{\text {highest }}$ and threshold voltage shift of lower state. As shown in Fig. 4, the lines representing relationships between threshold voltage shift of $P_{\text {highest }}$ and threshold voltage shift of lower states were fitted by the 3D NAND Flash experimental data. Similarly, the linear functions shown in Fig. 4 can also be designed as lookup tables stored in ROM in an actual engineering application.

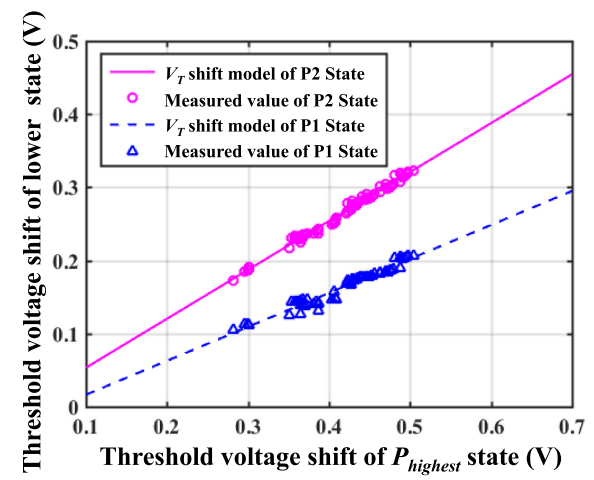

Fig. 4. Functional relationships between threshold voltage shift of $P_{\text {highest }}$ state and threshold voltage shift of lower state

\section{Experiment result of the novel valley search algorithm}

According to the functional relationship established in section 2 and section 3, the threshold voltage shift of P3 state, P2 state and P1 state can be calculated by the numeric size of $B$ and $C$ obtained by two read operations. The error of the mathematical model established in section 2 and section 3 can be calculated by actual threshold voltage shift minus threshold voltage shift determined by the novel valley search algorithm. Fourteen times experiments have been done in order to evaluate the error of the mathematical model. Referring to Fig. 5(a), the minimum error among these different tests is $0.6 \mathrm{mV}$, and the maximum error among these different tests is $31.0 \mathrm{mV}$.

Fourteen times experiments of actual read retry operations have been done by using the threshold voltage distribution valley found by the mathematical model established in the section 2 and 3. Fig. 5(b) shows the normalized raw bit error rates (RBERs) before read retry operations and after read retry operations and it was normalized by dividing the maximum value in the data. In other words, $100 \%$ represent the maximum bit error rate before read retry. After one years storage time at $55^{\circ} \mathrm{C}$, as Fig. 5(b) shows, raw bit error rate of the 3D NAND Flash Memory die with $3 \mathrm{~K} \mathrm{P} / \mathrm{E}$ cycles exceeds the error correction capability of the $\mathrm{BCH}(9088,8192,64)$ code far away. After read retry operation by using the novel valley search algorithm, raw bit error rate is reduced to $0.0344 \%$ and within $4.41 \%$ of the $\mathrm{BCH}(9088,8192,64)$ error correcting code (ECC) error correction capability. 
Specifically, $0.0344 \%$ is an average bit error rate after read retry operations and it is a true value.

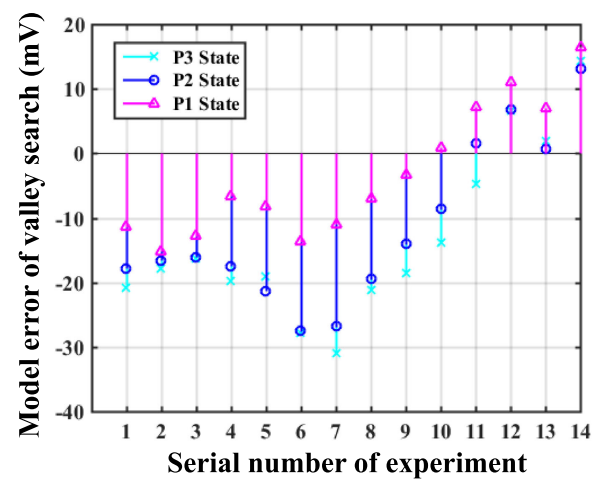

(a)

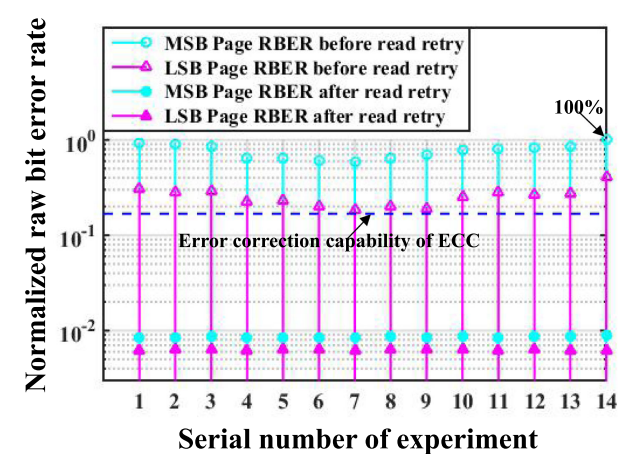

(b)

Fig. 5. Experiment result of the novel valley search algorithm

\section{Conclusion}

The novel valley search algorithm proposed in this paper can reduce read retry operations to two times. At the same time, the reading time can be also reduced dramatically. Experimental results show that the valley search algorithm has advanced performance in multi-level cell (MLC) NAND flash memories. With the development of multi-bit per cell storage technology, the mainstream of storage technology will transfer from multi-level cell to triple-level cell (TLC) and quadruple-level cell (QLC). Forseeable, the novel valley search algorithm proposed in this paper can also be extended to TLC and QLC engineering applications.

\section{Acknowledgments}

This work was supported by National Key Research and Development Program of China (No. 2018YFB1107700) and National Natural Science Foundation of China (No. 61474137). 